\title{
Anosov diffeomorphisms on infra-nilmanifolds modeled on a free 2-step nilpotent Lie group
}

\author{
Karel Dekimpe and Kelly Verheyen*
}

\begin{abstract}
In this article we study the existence question of Anosov diffeomorphisms on an infra-nilmanifold. After establishing a general existence criterion in terms of the associated holonomy representation, we concentrate on infra-nilmanifolds for which the covering Lie group is a free nilpotent Lie group. In turns out that in this case the criterion obtained before can be reduced drastically. Finally, we completely solve the existence question in case the covering Lie group is free 2-step nilpotent and the holonomy group is abelian.
\end{abstract}

Mathematics Subject Classification (2000). 37D20.

Keywords. Anosov diffeomorphism, infra-nilmanifold, nilpotent Lie group, almost Bieberbach group.

\section{Introduction}

In this article we will study Anosov diffeomorphisms $f$ on a closed manifold $M$. These Anosov diffeomorphisms have the property that there exists a continuous splitting of the tangent bundle $T M=E^{s} \oplus E^{u}$ of $M$ such that $d f$ is contracting on $E^{s}$ and expanding on $E^{u}$. In this way the tangent bundle splits into a stable part $E^{s}$ and an unstable part $E^{u}$ (see Definition 2.2 for a detailed definition).

Up till now, the only known examples of closed manifolds admitting an Anosov diffeomorphism are infra-nilmanifolds. Actually, it has been conjectured that this is the only class of manifolds in which one can expect to find Anosov diffeomorphisms.

It is well known (and rather easy to prove) that any torus of dimension at least two admits an Anosov diffeomorphism. There is also a very good description, due to Porteous [15], of which compact flat manifolds (these are the manifolds which are finitely covered by a torus) allow an Anosov diffeomorphism (see Theorem 5.1).

The situation for general nilmanifolds is much more difficult and the dimension alone does not provide any information on the existence question of an Anosov diffeomorphism. In fact, for any integer $n \geq 3$ one can easily give an example of a

\footnotetext{
${ }^{*}$ Ph.D. fellowship of the Research Foundation - Flanders (FWO).
} 
nilmanifold $M$ of dimension $n$ not admitting an Anosov diffeomorphism. Actually the requirement that a nilmanifold admits an Anosov diffeomorphism, seems to impose rather strong conditions on the nilmanifold. We refer the reader to [1], [2], [4], [6], [7], [8], [12], [13], [14] for some results about Anosov diffeomorphisms on nilmanifolds. The knowledge about Anosov diffeomorphisms on infra-nilmanifolds (manifolds which are finitely covered by nilmanifolds) is, except for the case of compact flat manifolds, almost non-existing. In this article we intend to present a first approach to the existence problem of Anosov diffeomorphisms on infra-nilmanifolds.

The class of infra-nilmanifolds we are going to study is the class of infra-nilmanifolds which are said to be modeled on a free nilpotent Lie group (see Section 5 for the definition). This is a natural class of manifolds to study, because it is known that the nilmanifolds which are finite covers of those infra-nilmanifolds do admit an Anosov diffeomorphism, provided that the dimension (or even better the number of generators of the corresponding free nilpotent Lie algebra) is big enough. This shows that the situation we are dealing with is as close as possible to the case of compact flat manifolds (and their finite covers, the tori).

The result of Porteous [15] on Anosov diffeomorphisms of flat manifolds mentioned above, gives a necessary and sufficient condition for the existence of an Anosov diffeomorphism in terms of the rational holonomy representation associated to such a flat manifold $M$. This holonomy representation is completely determined by the fundamental group $\pi_{1}(M)$, which fits in a short exact sequence

$$
1 \rightarrow \mathbb{Z}^{n} \rightarrow \pi_{1}(M) \rightarrow F \rightarrow 1
$$

where $\mathbb{Z}^{n}$ is a maximal abelian normal subgroup of $\pi_{1}(M)$ and $F$ is finite, and where the holonomy representation $\varphi: F \rightarrow \operatorname{Aut}\left(\mathbb{Z}^{n}\right) \subseteq \operatorname{Aut}\left(\mathbb{Q}^{n}\right)$ is induced by conjugation in $\pi_{1}(M)$.

In the third section of this article we show how this notion of a rational holonomy representation of a flat manifold can be generalized to the case of infra-nilmanifolds, where we obtain a representation $\varphi: F \rightarrow \operatorname{Aut}\left(\mathfrak{n}_{\mathbb{Q}}\right)$ of a finite group $F$ into the automorphism group of a Lie algebra $\mathfrak{n}_{\mathbb{Q}}$ over the rationals. This representation then induces what we call the abelianized rational holonomy representation $\bar{\varphi}: F \rightarrow \operatorname{Aut}\left(\frac{\mathfrak{n}_{\mathbb{Q}}}{\left[\mathfrak{n}_{\mathbb{Q}}, \mathfrak{n}_{\mathbb{Q}}\right]}\right)$.

In this article, we study the existence question of Anosov diffeomorphisms in terms of this (abelianized) rational holonomy representation. We remark that also for an infra-nilmanifold $M$, this associated abelianized rational holonomy representation is completely determined by the fundamental group $\pi_{1}(M)$.

First we deal with the general case of all infra-nilmanifolds and obtain the following criterium for the existence of Anosov diffeomorphisms:

Theorem A. Let $M$ be an infra-nilmanifold with associated rational holonomy representation $\varphi: F \rightarrow \operatorname{Aut}\left(N_{\mathbb{Q}}\right)$. Then $M$ admits an Anosov diffeomorphism if and only if there exists a hyperbolic automorphism (i.e., having no eigenvalues of mod- 
ulus 1) $\psi \in \operatorname{Aut}\left(\mathfrak{n}_{\mathbb{Q}}\right)$ with a normalized integer characteristic polynomial such that $\psi$ commutes with any element of $\varphi(F)$.

By a normalized integer polynomial $f(X) \in \mathbb{Q}[X]$, we mean a polynomial with integer coefficients and unit constant term (i.e., \pm 1 ).

Thereafter, we specialise to the class of infra-nilmanifolds modeled on a free nilpotent Lie group, where we are able to translate the previous result to a condition involving only the abelianized rational holonomy representation of such a manifold and we prove the following:

Theorem B. Let $M$ be an infra-nilmanifold modeled on a free c-step nilpotent Lie group and with abelianized rational holonomy representation $\bar{\varphi}: F \rightarrow \operatorname{Aut}\left(\frac{\mathfrak{n}_{\mathbb{Q}}}{\left[\mathfrak{n}_{\mathbb{Q}}, \mathfrak{n}_{\mathbb{Q}}\right]}\right)$. Then $M$ admits an Anosov diffeomorphism if and only if there exists an automorphism $\bar{\psi} \in \operatorname{Aut}\left(\frac{\mathfrak{n}_{\mathbb{Q}}}{\left[\mathfrak{n}_{\mathbb{Q}}, \mathfrak{n}_{\mathbb{Q}}\right]}\right)$ such that

(1) $\bar{\psi}$ has a normalized integer characteristic polynomial,

(2) $\bar{\psi}$ commutes with any element of $\bar{\varphi}(F)$ and

(3) if we denote the eigenvalues of $\bar{\psi}$ by $\lambda_{1}, \lambda_{2}, \ldots, \lambda_{n}$, then

$$
\left|\lambda_{j_{1}} \lambda_{j_{2}} \ldots \lambda_{j_{k}}\right| \neq 1
$$

for all $k \in\{1,2, \ldots, c\}$ and all $j_{1}, j_{2}, \ldots, j_{k} \in\{1,2, \ldots, n\}$.

It turns out that, as we restrict our attention to the case of infra-nilmanifolds modeled on a free 2-step nilpotent Lie group and with abelian holonomy group, we can reduce these conditions even more into a result which is very similar to the one Porteous obtained for compact flat manifolds:

Theorem C. Let $M$ be an infra-nilmanifold modeled on a free 2-step nilpotent Lie group, with abelian holonomy group $F$ and associated abelianized rational holonomy representation $\bar{\varphi}: F \rightarrow \operatorname{Aut}\left(\frac{N_{\mathbb{Q}}}{\left[N_{\mathbb{Q}}, N_{\mathbb{Q}}\right]}\right)$. Then $M$ admits an Anosov diffeomorphism if and only if

(1) each $\mathbb{Q}$-irreducible component $\bar{\varphi}_{i}$ of $\bar{\varphi}$ of multiplicity one splits in at least three components when seen as a representation over $\mathbb{R}$, and

(2) each $\mathbb{Q}$-irreducible component $\bar{\varphi}_{i}$ of $\bar{\varphi}$ of multiplicity two splits in more then one component when seen as a representation over $\mathbb{R}$.

As a conclusion of this article we formulate a conjecture, which is supported by the work in this article and also by some other experiments, which can really be seen as a very natural generalization of Porteous's result:

Conjecture. Let $M$ be an infra-nilmanifold modeled on a free c-step nilpotent Lie group, with holonomy group $F$ and associated abelianized rational holonomy representation $\bar{\varphi}: F \rightarrow \operatorname{Aut}\left(\frac{N_{\mathbb{Q}}}{\left[N_{\mathbb{Q}}, N_{\mathbb{Q}}\right]}\right)$. Then $M$ admits an Anosov diffeomorphism if 
and only if each $\mathbb{Q}$-irreducible component $\bar{\varphi}_{i}$ of $\bar{\varphi}$ of multiplicity $m$ splits in at least $c+2-m$ components when seen as a representation over $\mathbb{R}$.

\section{Infra-nilmanifolds and Anosov diffeomorphisms}

Before we can start a detailed study of Anosov diffeomorphisms on infra-nilmanifolds, we recall some basic material on those infra-nilmanifolds and their fundamental groups. We refer the reader to [3] and [11] and the references therein for more details.

Let $L$ be a connected and simply connected nilpotent Lie group and denote the group of continuous automorphisms of $L$ by $\operatorname{Aut}(L)$. Then we can form the semidirect product group $L \rtimes \operatorname{Aut}(L)$, which we will denote by $\operatorname{Aff}(L)$ and which acts on $L$ via

$$
(m, \alpha) \cdot n=m \alpha(n)
$$

for all $m, n \in L$ and all $\alpha \in \operatorname{Aut}(L)$. Fix a compact subgroup $C$ of $\operatorname{Aut}(L)$. A uniform and discrete subgroup $E$ of $L \rtimes C$ is called an almost-crystallographic group. Such an almost-crystallographic group $E$ acts properly discontinuously on $L$. In case $E$ is also torsion-free such a group is said to be an almost-Bieberbach group and then $E \backslash L$ is an infra-nilmanifold with fundamental group $E$.

If for an almost-Bieberbach group we have that $E \subset L$, then the quotient space $E \backslash L$ is a nilmanifold. In general, for any almost-crystallographic group $E$ one has that $N=E \cap L$ is a normal (even characteristic) subgroup of $E$ which is of finite index in $E$. Moreover, the group $N$ will be a uniform lattice of $L$ and $N$ is a maximal nilpotent subgroup of $E$. (In fact it is the unique normal and maximal nilpotent subgroup of $E$ ). So any almost-crystallographic group $E$ gives rise to a short exact sequence

$$
1 \rightarrow N \rightarrow E \rightarrow F \rightarrow 1
$$

in which $F$ is a finite group and $N$ is a finitely generated torsion-free nilpotent group which is maximal nilpotent in $E$. We will refer to such a short exact sequence (satisfying the conditions on $F$ and $N$ just mentioned) as being an essential extension. The group $F$ will be called the holonomy group of the almost-crystallographic group (and in case the group is an almost-Bieberbach group, we also talk about the holonomy group of the infra-nilmanifold).

Conversely, any group $E$ fitting in an essential extension (1) can be realized as an almost-crystallographic group. The connected and simply connected nilpotent Lie group $L$ needed for this realization is uniquely determined and is in fact the Mal'cev completion of $N$ (i.e., the unique connected and simply connected nilpotent Lie group $L$ containing $N$ as a uniform lattice). In the sequel, we will also use $N_{\mathbb{R}}$ to denote this Mal'cev completion of $N$.

In fact, the realization of an abstract group $E$ fitting in an essential extension has some strong uniqueness properties. This follows immediately from the following 
theorem of K. B. Lee and F. Raymond [11]:

Theorem 2.1. Let $L$ be a connected and simply connected nilpotent Lie group and let $E, E^{\prime} \subseteq \operatorname{Aff}(L)$ be two almost-crystallographic groups. Then for any isomorphism $\alpha: E \rightarrow E^{\prime}$ there exists an $(l, \varphi) \in \operatorname{Aff}(L)$ such that

$$
\alpha(e)=(l, \varphi) e(l, \varphi)^{-1} .
$$

We remark that this theorem has been generalized to morphisms $\alpha$ (not necessarily isomorphism) by K. B. Lee in [10], Theorem 1.1.

Let $E \subseteq \operatorname{Aff}(L)$ be an almost-Bieberbach group. If $(l, \varphi) \in \operatorname{Aff}(L)$ is such that $(l, \varphi) E(l, \varphi)^{-1}=E$, then the map

$$
f: L \rightarrow L, \quad x \mapsto l \varphi(x),
$$

induces a diffeomorphism on $E \backslash L$, which is referred to as an affine diffeomorphism of the infra-nilmanifold $E \backslash L$. Moreover, the induced isomorphism on the fundamental group is exactly the map $\alpha: E \rightarrow E, e \mapsto(l, \varphi) e(l, \varphi)^{-1}$. It follows that any homeomorphism of $E \backslash L$ is homotopic to a map induced from an affine diffeomorphism.

An automorphism $\varphi \in \operatorname{Aut}(L)$ is said to be hyperbolic if its differential $d \varphi \in$ $\operatorname{Aut}(\mathfrak{l})$, where $\mathfrak{l}$ is the Lie algebra corresponding to $L$, is a hyperbolic linear map (i.e., has no eigenvalues of modulus 1 ). An affine diffeomorphism of an infra-nilmanifold $E \backslash L$ is said to be a hyperbolic infra-nilmanifold automorphism if it is induced by an element $(l, \varphi)$, where $\varphi$ is a hyperbolic automorphism of $L$.

Hyperbolic infra-nilmanifold automorphisms play a crucial role in the study of Anosov diffeomorphisms.

Definition 2.2. A $C^{1}$-diffeomorphism $f: M \rightarrow M$ on a closed smooth manifold $M$ is said to be an Anosov diffeomorphism if there exists a continuous splitting $T M=E^{s} \oplus E^{u}$ of the tangent bundle of $M$ such that this splitting is $d f$-invariant and such that there exist a Riemannian metric $\|\cdot\|$ on $T M$ and real constants $c>0$ and $0<\lambda<1$ with

$$
\left\|d f^{n}(v)\right\| \leq c \lambda^{n}\|v\| \text { for } v \in E^{s} \quad \text { and } \quad\left\|d f^{n}(v)\right\| \geq c \lambda^{-n}\|v\| \text { for } v \in E^{u},
$$

for all positive integers $n$.

It is known that any hyperbolic infra-nilmanifold automorphism is an Anosov diffeomorphism of the infra-nilmanifold ([6], p. 63). Actually, by a theorem of A. Manning there is more:

Theorem 2.3 ([14]). Any Anosov diffeomorphism on an infra-nilmanifold is topologically conjugate to a hyperbolic infra-nilmanifold automorphism.

As a conclusion of this introduction we therefore have

Corollary 2.4. Let $M$ be an infra-nilmanifold. Then $M$ admits an Anosov diffeomorphism if and only if $M$ admits a hyperbolic infra-nilmanifold automorphism. 


\section{Rational realizations of almost-Bieberbach groups}

In the previous section we recalled that any group $E$ fitting in an essential extension (1) can be realized as a genuine almost-crystallographic subgroup of $\operatorname{Aff}\left(N_{\mathbb{R}}\right)$, where $N_{\mathbb{R}}$ is the Mal'cev completion of the unique normal and maximal nilpotent subgroup of $E$.

Actually, we can somehow strengthen this statement and use the radicable hull (or rational Mal'cev completion) of $N$. Let $N$ be any torsion-free and finitely generated nilpotent group. Then there exists a unique group $N_{\mathbb{Q}}$ such that $N_{\mathbb{Q}}$ is a torsion-free nilpotent radicable group containing $N$ as a subgroup and such that any element of $N_{\mathbb{Q}}$ has some positive power lying in $N$ (e.g., see [16], p. 107). In fact, if $N_{\mathbb{R}}$ is the Mal'cev completion of $N$ and $\mathfrak{n}_{\mathbb{R}}$ is the corresponding Lie algebra (over $\mathbb{R}$ ), then it is known that the exponential map exp: $\mathfrak{n}_{\mathbb{R}} \rightarrow N_{\mathbb{R}}$ is a diffeomorphism. If we denote the inverse of this map by $\log$, then $N_{\mathbb{Q}}$ can be defined as

$$
N_{\mathbb{Q}}=\exp (\mathbb{Q} \log (N)) .
$$

In fact, if we define $\mathfrak{n}_{\mathbb{Q}}=\mathbb{Q} \log (N)$ to be the $\mathbb{Q}$-span of $\log (N)$, then $\mathfrak{n}_{\mathbb{Q}}$ is a Lie algebra over the rationals and $\exp \left(\mathfrak{n}_{\mathbb{Q}}\right)=N_{\mathbb{Q}}$.

Suppose that two uniform discrete subgroups $N_{1}$ and $N_{2}$ of a given connected and simply connected nilpotent Lie group are given which are commensurable (i.e., their intersection $N_{1} \cap N_{2}$ is of finite index in both $N_{1}$ and $N_{2}$ ), then their radicable hulls are equal:

$$
\exp \left(\mathbb{Q} \log \left(N_{1}\right)\right)=\exp \left(\mathbb{Q} \log \left(N_{2}\right)\right)=\exp \left(\mathbb{Q} \log \left(N_{1} \cap N_{2}\right)\right) .
$$

Conversely, if $N_{1}$ and $N_{2}$ are two finitely generated subgroups of $N_{\mathbb{Q}}$ with $N_{\mathbb{Q}}=$ $\exp \left(\mathbb{Q} \log \left(N_{1}\right)\right)=\exp \left(\mathbb{Q} \log \left(N_{2}\right)\right)$ then $N_{1}$ and $N_{2}$ are commensurable and they are both uniform discrete subgroups of the connected and simply connected nilpotent Lie group $N_{\mathbb{R}}$. As a kind of shorthand we introduce the following definition.

Definition 3.1. Let $N_{\mathbb{Q}}$ be the radicable hull of a finitely generated torsion-free nilpotent group N. A subgroup $H$ of $N_{\mathbb{Q}}$ is said to be a full subgroup of $N_{\mathbb{Q}}$ if $H$ is finitely generated and $N_{\mathbb{Q}}$ is the radicable hull of $H$ (i.e., $N_{\mathbb{Q}}=\exp (\mathbb{Q} \log (H))$ ).

It follows that $N$ is a full subgroup of $N_{\mathbb{Q}}$ and for any other subgroup $H$ of $N_{\mathbb{Q}}$, we have that $H$ is a full subgroup of $N_{\mathbb{Q}}$ if and only if $N$ and $H$ are commensurable.

It is well known that any automorphism $\varphi$ of $N$ extends uniquely to an automorphism $\tilde{\varphi}$ of $N_{\mathbb{Q}}$ and any automorphism of $N_{\mathbb{Q}}$ induces a Lie algebra automorphism $\bar{\varphi}$ of $\mathfrak{n}_{\mathbb{Q}}$ such that

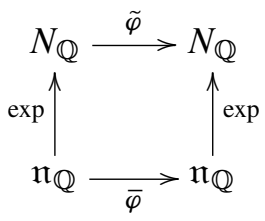


commutes. Conversely, for any automorphism $\bar{\varphi}$ of $\mathfrak{n}_{\mathbb{Q}}$ there exists an automorphism $\tilde{\varphi}$ of $N_{\mathbb{Q}}$ making the above diagram commutative. (The same holds when we replace $N_{\mathbb{Q}}$ by $N_{\mathbb{R}}$ and consider continuous automorphisms of $N_{\mathbb{R}}$.)

It follows that we can talk about the eigenvalues resp. the characteristic polynomial of an automorphism of $N$ (or $N_{\mathbb{Q}}$ ) by which we will mean the eigenvalues resp. characteristic polynomial of the corresponding linear automorphism of $\mathfrak{n}_{\mathbb{Q}}$ (or equivalently $\mathfrak{n}_{\mathbb{R}}$ ).

Using this terminology we can see that any automorphism of $N$ has a normalized integer characteristic polynomial. The converse is not true in general, i.e., there exist automorphisms of $N_{\mathbb{Q}}$ having a normalized integer characteristic polynomial, which do not restrict to an automorphism of $N$. There is however a partial converse, which will be sufficient for our purposes:

Theorem 3.2 ([4], Theorem 3.4). Let $N_{\mathbb{Q}}$ be the radicable hull of a finitely generated torsion-free nilpotent group $N$ and let $\varphi \in \operatorname{Aut}\left(N_{\mathbb{Q}}\right)$ be an automorphism with normalized integer characteristic polynomial. Then there exists a positive integer $k>0$ such that $\varphi^{k} \in \operatorname{Aut}(N)$.

Let us again focus on an almost-Bieberbach group $E$ and recall the notion of a rational realization as developed in [5]. As $E$ is an almost-Bieberbach group it fits in an essential extension $1 \rightarrow N \rightarrow E \rightarrow F \rightarrow 1$. Using the fact that any automorphism of $N$ has a unique lift to an automorphism of $N_{\mathbb{Q}}$ it is possible to construct the following commutative diagram of groups:

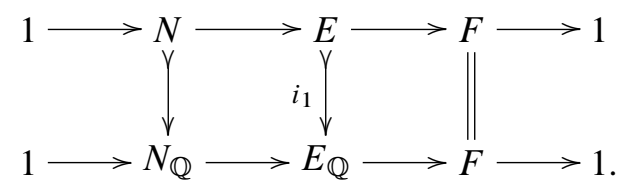

By using the same ideas of [3], Lemma 3.1.2 (using the radicable hull instead of the Mal'cev completion), one can prove that the bottom extension splits. So we can fix a splitting morphism $s: F \rightarrow E_{\mathbb{Q}}$ and we use $\varphi: F \rightarrow \operatorname{Aut}\left(N_{\mathbb{Q}}\right)$ to denote the induced morphism $\left(\varphi(f)(n)=s(f) n s(f)^{-1}\right)$. We will refer to the map $\varphi$ as being the rational holonomy representation determined by $E$. Actually, the rational holonomy representation of an almost-Bieberbach group $E$ is not uniquely determined (since the splitting $s$ is not), but any two rational holonomy representations $\varphi, \varphi^{\prime}: F \rightarrow \operatorname{Aut}\left(N_{\mathbb{Q}}\right)$ do induce the same maps $p \circ \varphi=p \circ \varphi^{\prime}$, where $p: \operatorname{Aut}\left(N_{\mathbb{Q}}\right) \rightarrow \operatorname{Out}\left(N_{\mathbb{Q}}\right)=\operatorname{Aut}\left(N_{\mathbb{Q}}\right) / \operatorname{Inn}\left(N_{\mathbb{Q}}\right)$ is the natural projection:

$$
p \circ \varphi=p \circ \varphi^{\prime}: F \rightarrow \operatorname{Aut}\left(N_{\mathbb{Q}}\right) \stackrel{p}{\rightarrow} \operatorname{Out}\left(N_{\mathbb{Q}}\right) .
$$

So we have that $E_{\mathbb{Q}} \cong N_{\mathbb{Q}} \rtimes F$. Moreover any $\varphi(f)$ can also be viewed as an 
automorphism of $N_{\mathbb{R}}$, and therefore we obtain the extended commutative diagram

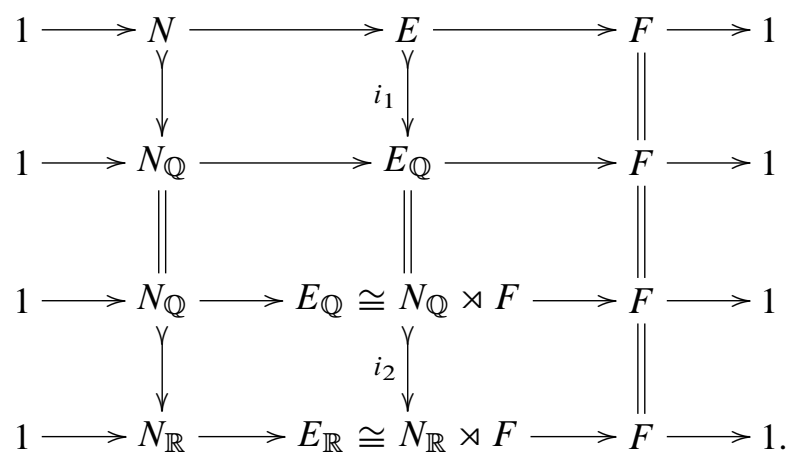

The composition $i=i_{2} \circ i_{1}: E \rightarrow N_{\mathbb{R}} \rtimes F \subseteq N_{\mathbb{R}} \rtimes \operatorname{Aut}\left(N_{\mathbb{R}}\right)$ realizes $E$ as a genuine almost-Bieberbach group, with the extra properties that for any element $e \in E$ we have that $i(e)=\left(n_{e}, f_{e}\right) \in \operatorname{Aff}\left(N_{\mathbb{R}}\right)$, where $n_{e} \in N_{\mathbb{Q}}$ and $f_{e}$ is an automorphism of $N_{\mathbb{R}}$ restricting to an automorphism of $N_{\mathbb{Q}}$. Indeed the restriction of $f_{e}$ to $N_{\mathbb{Q}}$ belongs to the image of the rational holonomy representation $\varphi(F)$ of $F$.

Definition 3.3. Let $E$ be an almost-crystallographic group fitting in an essential extension $1 \rightarrow N \rightarrow E \rightarrow F \rightarrow 1$. A rational realization of $E$ is an embedding $i: E \rightarrow \operatorname{Aff}\left(N_{\mathbb{R}}\right)$ factoring through $N_{\mathbb{Q}} \rtimes \operatorname{Aut}\left(N_{\mathbb{Q}}\right)$ via a map $i^{\prime}$,

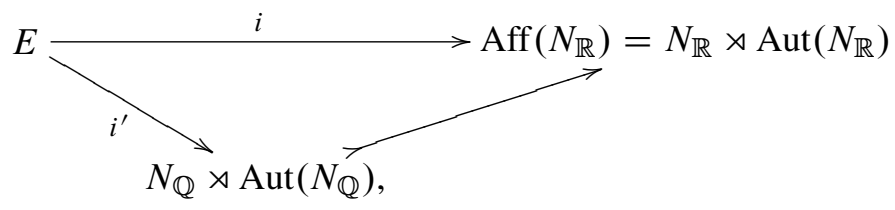

and such that $n=i^{\prime}(n)=i(n)$ for all $n \in E$.

We have just proved:

Lemma 3.4. Any almost-Bieberbach group E admits a rational realization.

It is also possible to sharpen K. B. Lee and F. Raymond's result with respect to a rational realization:

Remark 3.5. Let $E$ be an almost-Bieberbach group fitting in an essential extension $1 \rightarrow N \rightarrow E \rightarrow F \rightarrow 1$ and assume that $i: E \rightarrow \operatorname{Aff}\left(N_{\mathbb{R}}\right)$ is a rational realization of $E$. Then for any automorphism $\alpha \in \operatorname{Aut}(E)$ there exists an element $(l, \psi) \in \operatorname{Aff}\left(N_{\mathbb{R}}\right)$ such that

(1) $l \in N_{\mathbb{Q}}$,

(2) $\psi\left(N_{\mathbb{Q}}\right)=N_{\mathbb{Q}}$, 
(3) $\alpha(e)=(l, \psi) e(l, \psi)^{-1}$ for all $e \in E$.

Proof. The proof of this remark can be obtained by adapting the proof of K. B. Lee and F. Raymond to the case of radicable hulls (instead of using Mal'cev completions). See [11], Section 2.2 in [3], or [9].

\section{Anosov diffeomorphisms on infra-nilmanifolds}

In the previous section we have developed the necessary background to state and prove Theorem A, describing a necessary and sufficient condition which an infranilmanifold has to satisfy in order to admit an Anosov diffeomorphism.

Proof of Theorem A. Without loss of generality we may assume that we have fixed a rational realization $i: E \rightarrow \operatorname{Aff}\left(N_{\mathbb{R}}\right)$ and that $M$ is the quotient manifold $i(E) \backslash N_{\mathbb{R}}$. From now on we identify $E$ with $i(E)$.

First assume that $M$ admits an Anosov diffeomorphism $f$. By a result of Manning ([14], see Theorem 2.3), we know that $f$ is topologically conjugate to a hyperbolic infra-nilmanifold automorphism determined by some element $(l, \psi) \in \operatorname{Aff}\left(N_{\mathbb{R}}\right)$. By Remark 3.5, we may assume that $\psi\left(N_{\mathbb{Q}}\right)=N_{\mathbb{Q}}$ and $l \in N_{\mathbb{Q}}$ and we have that $(l, \psi) E(l, \psi)^{-1}=E$. Now a general element of $E$ can be written in the form $e=\left(n_{e}, f_{e}\right)$, with $n_{e} \in N_{\mathbb{Q}}$ and $f_{e} \in \operatorname{Aut}\left(N_{\mathbb{Q}}\right)$ (for ease of notation we regard $\operatorname{Aut}\left(N_{\mathbb{Q}}\right)$ as being a subgroup of $\operatorname{Aut}\left(N_{\mathbb{R}}\right)$, by identifying any element of $\operatorname{Aut}\left(N_{\mathbb{Q}}\right)$ with its unique continuous extension to $N_{\mathbb{R}}$ ). Note that

$$
\varphi(F)=\left\{f_{e} \mid e \in E\right\} .
$$

From $(l, \psi) E(l, \psi)^{-1}=E$ we find that

$$
\begin{aligned}
(l, \psi)\left(n_{e}, f_{e}\right)(l, \psi)^{-1} & =\left(l \psi\left(n_{e}\right), \psi f_{e}\right)\left(\psi^{-1}\left(l^{-1}\right), \psi^{-1}\right) \\
& =\left(l \psi\left(n_{e}\right) \psi\left(f_{e}\left(\psi^{-1}\left(l^{-1}\right)\right)\right), \psi f_{e} \psi^{-1}\right) \in E .
\end{aligned}
$$

It follows that for any $e \in E$, there exists an $e^{\prime} \in E$ for which $\psi f_{e} \psi^{-1}=f_{e^{\prime}}$. This means that $\psi$ normalizes $\varphi(F)$. Since $\varphi(F)$ is a finite subgroup of $\operatorname{Aut}\left(N_{\mathbb{Q}}\right)$, there must exist some power of $\psi$ which centralizes $\varphi(F)$. So by replacing $f$ (and then also $(l, \psi))$ by some positive power if needed, we may assume that $\psi$ commutes with any element of $\varphi(F)$.

Now we consider elements $(n, 1) \in N$. As $N$ is a characteristic subgroup of $E$, we must have that

$$
(l, \psi)(n, 1)(l, \psi)^{-1}=\left(l \psi(n) l^{-1}, 1\right) \in N .
$$

Let $\mu(l): N_{\mathbb{Q}} \rightarrow N_{\mathbb{Q}}, n \mapsto \ln l^{-1}$. Then the above computation shows that the composition $\mu(l) \circ \psi \in \operatorname{Aut}(N)$ and hence has a normalized integer characteristic 
polynomial. However, it is not hard to show that $\psi$ and $\mu(l) \circ \psi$ have the same characteristic polynomial, hence we have found a hyperbolic automorphism $\psi \in$ $\operatorname{Aut}\left(N_{\mathbb{Q}}\right)$ having a normalized integer characteristic polynomial and such that $\psi$ commutes with any element of $\varphi(F)$.

Conversely, assume that there is a hyperbolic automorphism $\psi \in \operatorname{Aut}\left(N_{\mathbb{Q}}\right)$ having a normalized integer characteristic polynomial such that $\psi$ commutes with any element of $\varphi(F)$. Then, by applying Theorem 3.2 and by replacing $\psi$ by a positive power if needed, we may assume that $\psi(N)=N$. As $F=E / N$ is finite, we can fix a finite number of elements

$$
\left(n_{1}, f_{1}\right) \in E, \quad\left(n_{2}, f_{2}\right) \in E, \ldots, \quad\left(n_{k}, f_{k}\right) \in E
$$

such that any element $e=\left(n_{e}, f_{e}\right)$ of $E$ can be written as a product

$$
e=(n, 1)\left(n_{i}, f_{i}\right)
$$

for some $n \in N_{\mathbb{Q}}$ and some $i \in\{1,2, \ldots, k\}$. Let $N_{1}$ be the subgroup of $N_{\mathbb{Q}}$ generated by $N$ and the elements $n_{1}, n_{2}, \ldots, n_{k}$. Then $N_{1}$ will be a full subgroup of $N_{\mathbb{Q}}$ containing $N$ as a subgroup of finite index. It follows that we can choose a subgroup $N_{2} \subseteq N$ which is normal in $N_{1}$ and of finite index in $N_{1}$. As $N_{2}$ is also a full subgroup of $N_{\mathbb{Q}}$, we can apply Theorem 3.2 (first for $N_{1}$ and then once again for $N_{2}$ ) and conclude, by replacing $\psi$ by some power if needed, that

$$
\psi(N)=N, \quad \psi\left(N_{1}\right)=N_{1} \quad \text { and } \quad \psi\left(N_{2}\right)=N_{2} .
$$

It follows that $\psi$ induces an automorphism of the finite group $N_{1} / N_{2}$, so some positive power of $\psi$ will induce the identity on $N_{1} / N_{2}$. So again after replacing $\psi$ with some power of it, we may assume that

$$
\text { for all } m \in N_{1} \text { there exists } n_{m} \in N_{2} \text { such that } \psi(m)=n_{m} m \text {. }
$$

We claim that $(1, \psi)$ induces a hyperbolic infra-nilmanifold automorphism on $M$. To check this, we show that $(1, \psi) E(1, \psi)^{-1}=E$. So take any element $e=$ $(n, 1)\left(n_{i}, f_{i}\right)$ of $E$, then

$$
\begin{aligned}
(1, \psi)(n, 1)\left(n_{i}, f_{i}\right)(1, \psi)^{-1} & =(\psi(n), 1)\left(\psi\left(n_{i}\right), f_{i}\right) \\
& =(\psi(n), 1)\left(n_{n_{i}} n_{i}, f_{i}\right) \quad\left(\text { with } n_{n_{i}} \in N_{2} \subseteq N\right) \\
& =(\psi(n), 1))\left(n_{n_{i}}, 1\right)\left(n_{i}, f_{i}\right) \\
& =\left(\psi(n) n_{n_{i}}, 1\right)\left(n_{i}, f_{i}\right) \\
& =\left(n^{\prime}, 1\right)\left(n_{i}, f_{i}\right) \in E \quad\left(\text { with } n^{\prime}=\psi(n) n_{n_{i}} \in N\right) .
\end{aligned}
$$

This shows that $(1, \psi) E(1, \psi)^{-1} \subseteq E$. In the same way one proves that $(1, \psi)^{-1} E(1, \psi) \subseteq E$, from which we conclude that $(1, \psi) E(1, \psi)^{-1}=E$. 


\section{Infra-nilmanifolds modeled on a free nilpotent Lie group}

Let $E$ be an almost-Bieberbach group inducing an essential extension $1 \rightarrow N \rightarrow$ $E \rightarrow F \rightarrow 1$. Recall that the corresponding infra-nilmanifold $M$ is then obtained as a quotient space $E \backslash N_{\mathbb{R}}$. We say that $M$ is modeled on the Lie group $N_{\mathbb{R}}$. A connected and simply connected nilpotent Lie group $L$ is said to be free nilpotent of class $c$ (with $k$ generators) if and only if the corresponding Lie algebra $\mathfrak{l}$ is free nilpotent of class $c$ (with $k$ generators). So $\mathbb{R}^{k}$ is the free nilpotent Lie group of class 1 on $k$ generators.

The work of Porteous ([15]), completely describes which flat Riemannian manifolds do admit an Anosov diffeomorphism and this in terms of the rational holonomy representation. Let us recall the main result of that paper.

Theorem 5.1 ([15], Theorem 6.1). Let $M$ be a flat manifold with associated Bieberbach group $E$ fitting in an essential extension $0 \rightarrow \mathbb{Z}^{n} \rightarrow E \rightarrow F \rightarrow 1$ and with an induced rational holonomy representation $\varphi: F \rightarrow \operatorname{GL}(n, \mathbb{Q})=\operatorname{Aut}\left(\mathbb{Q}^{n}\right)$. Then $M$ admits an Anosov diffeomorphism if and only if each $\mathbb{Q}$-irreducible component of the representation $\varphi$ which is of multiplicity one is reducible over $\mathbb{R}$.

The flat Riemannian manifolds are exactly those infra-nilmanifolds which are modeled on a Lie group $\mathbb{R}^{k}$, i.e., on a free nilpotent Lie group of class 1 . Our aim is to extend Porteous' result to infra-nilmanifolds modeled on a free nilpotent Lie group.

As a first step in this direction we will prove a result in the same spirit as Theorem A, obtaining a necessary condition which is easier to check then the one in Theorem A. Thereafter we will be able to adapt this criterium into a necessary and sufficient condition for the class of infra-nilmanifolds modeled on a free nilpotent Lie group, too.

In order to be able to formulate this new result let us introduce the following notion.

Definition 5.2. Let $E$ be an almost-Bieberbach group with associated rational holonomy representation $\varphi: F \rightarrow \operatorname{Aut}\left(N_{\mathbb{Q}}\right)$. The abelianized rational holonomy representation is the induced map

$$
\bar{\varphi}: F \rightarrow \operatorname{Aut}\left(\frac{N_{\mathbb{Q}}}{\left[N_{\mathbb{Q}}, N_{\mathbb{Q}}\right]}\right) \text { with } \bar{\varphi}\left(n\left[N_{\mathbb{Q}}, N_{\mathbb{Q}}\right]\right)=\varphi(n)\left[N_{\mathbb{Q}}, N_{\mathbb{Q}}\right] .
$$

Remark 5.3. (1) $\frac{N_{\mathbb{Q}}}{\left[N_{\mathbb{Q}}, N_{\mathbb{Q}}\right]}$ is an abelian group (and a rational vector space) and is actually isomorphic $\left(\log\right.$ induces a linear isomorphism) to $\frac{\mathfrak{n}_{\mathbb{Q}}}{\left[\mathfrak{n}_{\mathbb{Q}}, n_{\mathbb{Q}}\right]}$. So we can equally well speak about the abelianized rational holonomy representation

$$
\bar{\varphi}: F \rightarrow \operatorname{Aut}\left(\frac{\mathfrak{n}_{\mathbb{Q}}}{\left[\mathfrak{n}_{\mathbb{Q}}, \mathfrak{n}_{\mathbb{Q}}\right]}\right) .
$$


(2) There is still another way of viewing this abelianized holonomy representation. Starting from the short exact sequence

$$
1 \rightarrow N \rightarrow E \rightarrow F \rightarrow 1,
$$

we have the induced short exact sequence of groups

$$
1 \rightarrow \frac{N}{[N, N]} \rightarrow \frac{E}{[N, N]} \rightarrow F \rightarrow 1,
$$

inducing a representation $\tilde{\varphi}: F \rightarrow \operatorname{Aut}\left(\frac{N}{[N, N]}\right)$ by conjugation inside $\frac{E}{[N, N]}$. Now we can form the composition

$$
F \stackrel{\tilde{\varphi}}{\rightarrow} \operatorname{Aut}\left(\frac{N}{[N, N]}\right) \rightarrow \operatorname{Aut}\left(\frac{N}{[N, N]} \otimes \mathbb{Q}\right) .
$$

This composition is another way to represent $\bar{\varphi}$ (note that $\left.\frac{N}{[N, N]} \otimes \mathbb{Q} \cong \frac{N_{\mathbb{Q}}}{\left[N_{\mathbb{Q}}, N_{\mathbb{Q}}\right]}\right)$.

As an immediate consequence of Theorem A we find

Proposition 5.4. Let $E$ be an almost-Bieberbach group with abelianized rational holonomy representation $\bar{\varphi}: F \rightarrow \operatorname{Aut}\left(\frac{N_{\mathbb{Q}}}{\left[N_{\mathbb{Q}}, N_{\mathbb{Q}}\right]}\right)$. If the infra-nilmanifold $M$ corresponding to $E$ admits an Anosov diffeomorphism, then there exists a hyperbolic $\bar{\psi} \in \operatorname{Aut}\left(\frac{N_{\mathbb{Q}}}{\left[N_{\mathbb{Q}}, N_{\mathbb{Q}}\right]}\right)$ commuting with any element of $\bar{\varphi}(F)$ and such that $\bar{\psi}$ has a normalized integer characteristic polynomial.

Proof. By Theorem A we know that there exists a hyperbolic $\psi \in \operatorname{Aut}\left(\mathfrak{n}_{\mathbb{Q}}\right)$ commuting with any element of $\varphi(F)$, where $\varphi$ is a rational holonomy representation and such that $\psi$ has a normalized integer characteristic polynomial. It follows that $\psi$ induces an automorphism $\bar{\psi}$ of $\frac{N_{\mathbb{Q}}}{\left[N_{\mathbb{Q}}, N_{\mathbb{Q}}\right]}$, which is also hyperbolic and commutes with any element of $\bar{\varphi}(F)$. Moreover, the characteristic polynomial of $\bar{\psi}$ is a polynomial with rational coefficients and is a factor of the characteristic polynomial of $\psi$. It follows that this characteristic polynomial has integer coefficients and unit constant term as claimed.

It is easy to see that the converse of the above proposition does not hold. However, for infra-nilmanifolds modeled on a free nilpotent Lie group, we can adapt the above proposition and obtain Theorem B, giving us a necessary and sufficient condition for this type of infra-nilmanifolds to admit an Anosov diffeomorphism. But first we prove the following lemma.

Lemma 5.5. Let $\mathrm{g}$ be a finite dimensional nilpotent Lie algebra over $\mathbb{Q}$. Assume that $\varphi: \mathfrak{g} \rightarrow \mathfrak{g}$ is Lie algebra morphism and let $\bar{\varphi}: \mathfrak{g} /[\mathfrak{g}, \mathrm{g}] \rightarrow \mathfrak{g} /[\mathfrak{g}, \mathrm{g}]$ be the induced linear map. Then $\varphi$ will have a normalized integer characteristic polynomial if and only if $\bar{\varphi}$ has a normalized integer characteristic polynomial. 
Proof. As the characteristic polynomial $m_{\bar{\varphi}}(x)$ is a rational factor of the characteristic polynomial $m_{\varphi}(x)$, it follows that $m_{\bar{\varphi}}(x)$ has integer coefficients and unit constant term when $m_{\varphi}(x)$ has integer coefficients and unit constant term.

Now assume that $\bar{\varphi}$ has a normalized integer characteristic polynomial. Let us consider the graded Lie algebra $\mathfrak{g}_{\mathrm{gr}}$ which is associated to $\mathrm{g}$. Then

$$
\mathrm{g}_{\mathrm{gr}}=\bigoplus_{i=1}^{c} \mathrm{~g}_{i} \quad \text { with } \mathrm{g}_{i}=\gamma_{i}(\mathrm{~g}) / \gamma_{i+1}(\mathrm{~g}),
$$

and the Lie bracket on $g_{g r}$ is determined by

$$
\left[X+\gamma_{i+1}(\mathfrak{g}), Y+\gamma_{j+1}(\mathfrak{g})\right]=[X, Y]+\gamma_{i+j+1} \in \mathfrak{g}_{i+j}
$$

for all $X \in \gamma_{i}(\mathrm{~g})$ and all $Y \in \gamma_{j}(\mathrm{~g})$.

The morphism $\varphi$ induces an automorphism $\varphi_{\mathrm{gr}}$ of $\mathrm{g}_{\mathrm{gr}}$ by $\varphi_{\mathrm{gr}}\left(X+\gamma_{i+1}(\mathrm{~g})\right)=$ $\varphi(X)+\gamma_{i+1}(\mathfrak{g})$ for all $X \in \gamma_{i}(\mathfrak{g})$. Note that $\varphi_{\mathrm{gr}}\left(\mathfrak{g}_{i}\right)=\mathfrak{g}_{i}$ and that $\varphi$ and $\varphi_{\mathrm{gr}}$ have the same characteristic polynomial.

Hence it is enough to show that the characteristic polynomial of $\varphi_{\mathrm{gr}}$ has integer coefficients and unit constant term. To prove this, consider the $k$-fold tensor product of $\bar{\varphi}$ :

$$
\otimes^{k} \bar{\varphi}: \stackrel{k}{\bigotimes} \mathfrak{g}_{1} \rightarrow \stackrel{k}{\bigotimes}^{\mathfrak{g}_{1}}
$$

If the collection of eigenvalues of $\bar{\varphi}$ is $\lambda_{1}, \lambda_{2}, \ldots, \lambda_{n}$, each listed as many times as its multiplicity, then the collection of eigenvalues of $\otimes^{k} \varphi$ consists of all products of the form

$$
\lambda_{i_{1}} \lambda_{i_{2}} \ldots \lambda_{i_{k}}, \quad 1 \leq i_{1}, i_{2}, \ldots, i_{k} \leq n .
$$

So the characteristic polynomial of $\otimes^{k} \bar{\varphi}$ will be

$$
\prod_{1 \leq i_{1}, i_{2}, \ldots, i_{k} \leq n}\left(x-\lambda_{i_{1}} \lambda_{i_{2}} \cdots \lambda_{i_{k}}\right),
$$

which is symmetric in the $\lambda_{j_{i}}$ and hence will be a normalized integer polynomial.

Let

$$
T\left(g_{1}\right)=\bigoplus_{n=0}^{\infty} \otimes^{n} g_{1}
$$

be the tensor algebra of $g_{1}$ and let

$$
T^{(k)}\left(\mathfrak{g}_{1}\right)=\bigoplus_{n=k}^{\infty} \bigotimes^{n} \mathfrak{g}_{1} .
$$

From the discussion above, we get that the map that $\bar{\varphi}$ induces on the truncated tensor algebra

$$
T_{c}\left(\mathfrak{g}_{1}\right)=T\left(\mathfrak{g}_{1}\right) / T^{(c+1)}\left(\mathfrak{g}_{1}\right)
$$

has a normalized integer characteristic polynomial. Now the free $c$-step nilpotent Lie algebra, say $\mathfrak{f}_{c}\left(\mathfrak{g}_{1}\right)$, on $\mathfrak{g}_{1}$ is the Lie subalgebra of $T_{c}\left(\mathfrak{g}_{1}\right)$ generated (as a Lie 
algebra) by $\mathfrak{g}_{1}$. It follows that $\bar{\varphi}$ induces a map, say $\tilde{\bar{\varphi}}$, on $\mathfrak{f}_{c}\left(\mathfrak{g}_{1}\right)$ whose characteristic polynomial has integer coefficients and unit constant term.

Moreover, the graded Lie algebra $\mathfrak{g}_{\mathrm{gr}}$ can be obtained as a quotient $\mathfrak{f}_{c}\left(\mathfrak{g}_{1}\right) / I$, where $I$ is an ideal of $\mathfrak{f}_{c}\left(g_{1}\right)$ and $I$ is invariant under $\tilde{\bar{\varphi}}$. We have that the map that $\tilde{\bar{\varphi}}$ induces on the quotient $\mathfrak{f}_{c}\left(g_{1}\right) / I$ has a normalized integer characteristic polynomial. Since this induced map is exactly the map $\varphi_{\mathrm{gr}}$, the proof of the lemma is finished.

We are now ready to prove Theorem B.

Proof of Theorem B. As $M$ is modeled on a free $c$-step nilpotent Lie group, we know that the Lie algebra $\mathfrak{n}_{\mathbb{R}}$ and hence also $\mathfrak{n}_{\mathbb{Q}}$ is a free $c$-step nilpotent Lie algebra.

First assume that $M$ admits an Anosov diffeomorphism. By Theorem A, we know that there exists a hyperbolic $\psi \in \operatorname{Aut}\left(\mathfrak{n}_{\mathbb{Q}}\right)$ commuting with any element of $\varphi(F)$ (where $\varphi$ is a rational holonomy representation) and such that $\psi$ has a normalized integer characteristic polynomial. As already explained in the proof of Proposition 5.4, we obtain that $\bar{\psi}$ commutes with any element of $\bar{\varphi}(F)$ and also has a normalized integer characteristic polynomial. If we denote the eigenvalues of $\bar{\psi}$ by $\lambda_{1}, \lambda_{2}, \ldots, \lambda_{n}$, then the eigenvalues of $\psi$ are exactly the numbers of the form

$$
\lambda_{j_{1}} \lambda_{j_{2}} \ldots \lambda_{j_{k}}
$$

with $k \in\{1,2, \ldots, c\}$ and $j_{1}, j_{2}, \ldots, j_{k} \in\{1,2, \ldots, n\}$ except perhaps those with $k>1$ and $j_{1}=j_{2}=\cdots=j_{k}$. As $\psi$ is hyperbolic, we have that

$$
\left|\lambda_{j_{1}} \lambda_{j_{2}} \cdots \lambda_{j_{k}}\right| \neq 1
$$

Note that this also holds for the case $k>1$ and $j_{1}=j_{2}=\cdots=j_{k}$ because $\left|\lambda_{j_{1}}^{k}\right|=\left|\lambda_{j_{1}}\right|^{k} \neq 1$ and $\left|\lambda_{j_{1}}\right| \neq 1$. This finishes the proof of the first direction.

Now assume the existence of a $\bar{\psi}$ as claimed. As $F$ is a finite subgroup, there exists a set $X_{1}, X_{2}, \ldots, X_{n} \in \mathfrak{n}_{\mathbb{Q}}$ such that $\mathfrak{n}_{\mathbb{Q}}$ is the free $c$-step nilpotent Lie algebra on the generators $X_{1}, X_{2}, \ldots, X_{n}$ (i.e., the canonical images of the $X_{i}$ form a vector space basis of $\frac{\mathfrak{n}_{\mathbb{Q}}}{\left[\mathfrak{n}_{\mathbb{Q}}, \mathfrak{n}_{\mathbb{Q}}\right]}$ ) and such that if we use $\mathfrak{n}_{1}$ to denote the subspace of $\mathfrak{n}$ generated by $X_{1}, X_{2}, \ldots, X_{n}$, we have that $\varphi(f)\left(\mathfrak{n}_{1}\right)=\mathfrak{n}_{1}$ for any $f \in F$.

Now, for $k=2,3, \ldots, c$, we let $\mathfrak{n}_{k}$ be the subspace of $\mathfrak{n}$, which is generated (as a vector space) by all elements of the form $\left[X_{j_{1}},\left[X_{j_{2}}, \ldots,\left[X_{j_{k-1}}, X_{j_{k}}\right] \ldots\right]\right]$ and we let $\mathfrak{n}_{k}=0$ when $k>c$. Then

$$
\mathfrak{n}=\mathfrak{n}_{1} \oplus \mathfrak{n}_{2} \oplus \mathfrak{n}_{3} \oplus \cdots
$$

is a positive grading of $\mathfrak{n}$ (i.e., $\left[\mathfrak{n}_{i}, \mathfrak{n}_{j}\right] \subseteq \mathfrak{n}_{i+j}$ ) and $\varphi(f)$ respects this grading for any $f$ (i.e., $\varphi(f)\left(\mathfrak{n}_{i}\right)=\mathfrak{n}_{i}$ ). Of course, we have that $\mathfrak{n}_{1}$ is isomorphic to $\frac{\mathfrak{n}_{\mathbb{Q}}}{\left[\mathfrak{n}_{\mathbb{Q}}, \mathfrak{n}_{\mathbb{Q}}\right]}$ and after identifying these two spaces we can say that $\bar{\varphi}(f)=\varphi(f)_{\mid \mathfrak{n}_{1}}$. In the same way we can view $\bar{\psi}$ as being a linear automorphism of $\mathfrak{n}_{1}$. As $\mathfrak{n}$ is free $c$-step 
nilpotent, the map $\bar{\psi}$ (seen as a map on $\mathfrak{n}_{1}$ ) has a unique extension to a Lie algebra automorphism $\psi$ of $\mathfrak{n}_{\mathbb{Q}}$. Moreover, from the fact that $\bar{\psi}=\psi_{\mid \mathfrak{n}_{1}}$ commutes with $\bar{\varphi}(f)=\varphi(f)_{\mid \mathfrak{n}_{1}}$, one deduces immediately that $\psi$ commutes with $\varphi$. Moreover, the eigenvalues of $\psi$ will all be of the form

$$
\lambda_{j_{1}} \lambda_{j_{2}} \ldots \lambda_{j_{k}}
$$

with $k \in\{1,2, \ldots, c\}$ and $j_{1}, j_{2}, \ldots, j_{k} \in\{1,2, \ldots, n\}$, showing that $\psi$ is a hyperbolic automorphism. Finally, by Lemma 5.5 the characteristic polynomial of $\psi$ will have integer coefficients and unit constant term. The theorem is now proved by applying Theorem A.

\section{Rational representations of finite abelian groups}

Given an infra-nilmanifold $M$ (modeled on a free $c$-step nilpotent Lie group), Theorem B provides necessary and sufficient conditions on the associated abelianized rational holonomy representation $\bar{\varphi}$ for $M$ to admit an Anosov diffeomorphism. In this section we concentrate on the case of infra-nilmanifolds modeled on a free 2step nilpotent Lie group, and try to reduce the conditions even more. Therefore, we will have a closer look at rational representations of finite groups satisfying similar conditions as those mentioned in Theorem B.

Let $T: F \rightarrow \operatorname{GL}(n, \mathbb{Q})$ be a rational representation of a finite group $F$. Translating the conditions in Theorem $\mathrm{B}$, we want to know when there exists a matrix $C \in \mathrm{GL}(n, \mathbb{Q})$ commuting with every element of $T(F)$ such that $C$ has a normalized integer characteristic polynomial with no roots $\lambda, \mu$ where $|\lambda|=1$ or $|\lambda \mu|=1$.

We can reduce these conditions to conditions on the $\mathbb{Q}$-irreducible components of $T$, and we find that the only $\mathbb{Q}$-irreducible components that really matter are those which occur with multiplicity one or two.

Theorem 6.1. Let $T: F \rightarrow \mathrm{GL}(n, \mathbb{Q})$ be a representation of a finite group $F$ and write $\Phi=\operatorname{Im}(T)$. Then the following assertions are equivalent:

(1) There exists a matrix $C \in \mathrm{GL}(n, \mathbb{Q})$ that commutes with every element of $\Phi$, and such that $C$ has a normalized integer characteristic polynomial with no roots $\lambda$, $\mu$ where $|\lambda|=1$ or $|\lambda \mu|=1$.

(2) For every $\mathbb{Q}$-irreducible component $T_{i}: F \rightarrow \mathrm{GL}\left(n_{i}, \mathbb{Q}\right)$ of $T$ that occurs with multiplicity one resp. two, there exists a matrix $C_{i} \in \mathrm{GL}\left(n_{i}, \mathbb{Q}\right)$ resp. $C_{i} \in$ $\mathrm{GL}\left(2 n_{i}, \mathbb{Q}\right)$ that commutes with every element of $T_{i}(F)$ resp. $\left(T_{i} \oplus T_{i}\right)(F)$, and has a normalized integer characteristic polynomial without roots $\lambda, \mu$ with $|\lambda|=1$ or $|\lambda \mu|=1$.

Proof. First suppose that there exists a matrix $C \in \mathrm{GL}(n, \mathbb{Q})$ that commutes with every element of $\Phi$ and that satisfies the conditions. Now change the basis of $\mathbb{Q}^{n}$ 
such that $T$ splits up as $T=T_{1} \oplus \cdots \oplus T_{k}$ with each $T_{i} \mathbb{Q}$-irreducible and with equivalent $T_{i}$ identical and adjacent. Then if some $T_{i}$ occurs with multiplicity one, the new matrix $\widetilde{C}=P^{-1} C P$, where $P$ is the matrix of the new basis, will have a single block corresponding to $T_{i}$ and commuting with it. The characteristic polynomial of this block will divide the characteristic polynomial of $C$ and so it will satisfy the conditions. Equivalently, if some $T_{i}$ occurs with multiplicity two, $\widetilde{C}$ will have a block corresponding to $T_{i} \oplus T_{i}$ commuting with it and its characteristic polynomial will again satisfy the conditions.

Conversely, suppose that for every $\mathbb{Q}$-irreducible component $T_{i}$ of multiplicity one resp. two, we have a matrix $K_{i}$ commuting with $\operatorname{Im}\left(T_{i}\right)$ resp. $\operatorname{Im}\left(T_{i} \oplus T_{i}\right)$ and satisfying the conditions. We construct the matrix $\widetilde{C}$. If some $T_{i}$ has multiplicity one or two, put the corresponding $K_{i}$ in its appropriate place on the diagonal. Now if some $T_{i}$ has multiplicity three, four or five, put

$$
\left(\begin{array}{ccc}
I & I & I \\
I & 2 I & 2 I \\
I & 2 I & 3 I
\end{array}\right),\left(\begin{array}{cccc}
I & I & I & I \\
I & I & I & 2 I \\
I & I & 0 & 0 \\
I & 2 I & 0 & 0
\end{array}\right) \text { or resp. }\left(\begin{array}{ccccc}
I & I & I & I & I \\
I & 2 I & 2 I & 2 I & 2 I \\
I & 2 I & 3 I & 3 I & 3 I \\
I & 2 I & 3 I & 4 I & 4 I \\
I & 2 I & 3 I & 4 I & 5 I
\end{array}\right)
$$

in the appropriate place; if some $T_{i}$ has even higher multiplicity, we can use suitable combinations of these. So now we have different blocks in $\widetilde{C}$, whose eigenvalues satisfy the conditions. However, there might be blocks, say $K_{i}$ and $K_{j}$, such that $K_{i}$ has an eigenvalue $\lambda_{i}$ and $K_{j}$ has an eigenvalue $\lambda_{j}$ with $\left|\lambda_{i} \lambda_{j}\right|=1$, so problems might arise for our eigenvalues of $\widetilde{C}$. Anyway, we can easily solve this by taking a power of (one of) the blocks if necessary. Now $\widetilde{C}$ commutes with $\operatorname{Im}(T)$ referred to the new basis and by construction, $\widetilde{C}$ has no eigenvalues $\lambda, \mu$ with $|\lambda|=1$ or $|\lambda \mu|=1$ and the characteristic polynomial of $\widetilde{C}$ has integer coefficients. So if we write $C=P \widetilde{C} P^{-1}, C$ will commute with $\Phi$ and satisfy our conditions.

This theorem instructs us to have a closer look at $\mathbb{Q}$-irreducible rational representations.

From this moment onwards, we restrict our attention to representations of finite, abelian groups $F$.

Let $F$ be a finite abelian group and $T: F \rightarrow \mathrm{GL}(n, \mathbb{Q})$ a $\mathbb{Q}$-irreducible representation of $F$. Write $\Phi=\operatorname{Im}(T)$, then since $F$ is finite and abelian, so is $\Phi$, and we can see that $\Phi$ is an abelian subgroup of the multiplicative group of $\operatorname{End}_{F}\left(\mathbb{Q}^{n}\right)$. Thus by the following well-known theorem, we know that $\Phi$ is cyclic.

Theorem 6.2. Every finite, abelian subgroup of the multiplicative group of a division ring is cyclic.

We can choose a generator $M=T(f)$ of $\Phi$, whose characteristic polynomial will be $\mathbb{Q}$-irreducible by the following lemma, the proof of which is left to the reader. 
Lemma 6.3. A linear transformation $T$ on a finite-dimensional vector space over $\mathbb{Q}$ is $\mathbb{Q}$-reducible if and only if the characteristic polynomial of $T$ is reducible over $\mathbb{Q}$.

Since $M$ has finite order, it is diagonalizable and its eigenvalues are roots of unity. Suppose $\xi_{d}$ is a primitive $d$-th root of unity which is an eigenvalue of $M$. Then the minimal polynomial of $\xi_{d}$ over $\mathbb{Q}$ is $\phi_{d}(X)$ (the $d$-th cyclotomic polynomial), and since the characteristic polynomial of $M$ is $\mathbb{Q}$-irreducible, it needs to be exactly $\phi_{d}(X)$. So $M$ has order $d$, its characteristic polynomial is $\phi_{d} \in \mathbb{Z}[X]$, and the eigenvalues of $M$ are exactly the $\varphi(d)$ primitive $d$-th roots of unity (in which $\varphi$ denotes the Euler phi function); we also find that $\varphi(d)=n$. Write $K=\mathbb{Q}\left(\xi_{d}\right)$ and let $\Gamma(K / \mathbb{Q})=\left\{\sigma_{1}, \ldots, \sigma_{\varphi(d)}\right\}$ denote the Galois group of $K$ over $\mathbb{Q}$. Then $K$ is a minimal splitting field of $T$ and $\operatorname{Spec}(M)=\left\{\sigma_{1}\left(\xi_{d}\right), \ldots, \sigma_{\varphi(d)}\left(\xi_{d}\right)\right\}$, where every eigenvalue occurs with algebraic multiplicity one. So we can choose an eigenvector $v \in K^{n}$ with corresponding eigenvalue $\xi_{d}$, and then $\sigma_{i}(v)$ will be an eigenvector with corresponding eigenvalue $\sigma_{i}\left(\xi_{d}\right)$ for all $i$. So $\left\{\sigma_{1}(v), \ldots, \sigma_{n}(v)\right\}$ is linearly independent and generates $K^{n}$ over $K$. If we take $P \in \mathrm{GL}(n, K)$ whose columns are exactly those Galois-conjugate eigenvectors $\left\{\sigma_{1}(v), \ldots, \sigma_{n}(v)\right\}$, then

$$
P^{-1} M P=\left(\begin{array}{ccc}
\sigma_{1}\left(\xi_{d}\right) & \cdots & 0 \\
\vdots & \ddots & \vdots \\
0 & \cdots & \sigma_{n}\left(\xi_{d}\right)
\end{array}\right) \in \operatorname{GL}(n, K)
$$

is a diagonal matrix.

If we want to find $C \in \operatorname{GL}(n, \mathbb{Q})$ commuting with $M$, then $M$ and $C$ need to be simultaneously diagonalizable. So we know that $v$ is also an eigenvector of $C$, and if $\lambda$ is the corresponding eigenvalue, then $\operatorname{Spec}(C)=\left\{\sigma_{1}(\lambda), \ldots, \sigma_{n}(\lambda)\right\}$ (here not all $\sigma_{i}(\lambda)$ need to be different), and $\sigma_{i}(v)$ is an eigenvector of $C$ corresponding to the eigenvalue $\sigma_{i}(\lambda)$ for all $i$.

Since we want the characteristic polynomial and the eigenvalues of $C$ to satisfy the conditions in Theorem 6.1, we want to know when we can find an algebraic unit $\mu \in U_{K}$ (where $U_{K}$ denotes the group of the algebraic units in $K$, i.e., the units in the ring $\mathcal{O}_{K}$ of the algebraic integers in $K$ ) so that $\sigma_{1}(\mu), \ldots, \sigma_{n}(\mu)$ satisfy these conditions. We prove the following lemma:

Lemma 6.4. Let $K=\mathbb{Q}\left(\xi_{d}\right)$ be the algebraic extension of $\mathbb{Q}$, where $\xi_{d}$ is a primitive $d$-th root of unity, and let $\Gamma(K / \mathbb{Q})=\left\{\sigma_{1}, \ldots, \sigma_{\varphi(d)}\right\}$ denote the Galois group of $K$ over $\mathbb{Q}$. Then we can find an algebraic unit $\mu$ in $K$ satisfying $\left|\sigma_{i}(\mu)\right| \neq 1$ and $\left|\sigma_{i}(\mu) \sigma_{j}(\mu)\right| \neq 1$ for all $1 \leq i, j \leq \varphi(d)$ if and only if $\varphi(d)>4$.

Proof. For the proof of this lemma we are inspired by the construction of the isomorphism in the proof of Dirichlet's unit theorem (see [17]) (in our case, $s=0$ and $2 t=\varphi(d))$. 
Write $\Gamma(K / \mathbb{Q})=\left\{\sigma_{1}, \ldots, \sigma_{t}, \overline{\sigma_{1}}, \ldots, \overline{\sigma_{t}}\right\}$ and let $U_{K}$ denote the group of units in the ring of the algebraic integers in $K$. Now define the map

$$
l: U_{K} \rightarrow \mathbb{R}^{t}, \quad \alpha \mapsto\left(l_{1}(\alpha), \ldots, l_{t}(\alpha)\right)=\left(2 \log \left|\sigma_{1}(\alpha)\right|, \ldots, 2 \log \left|\sigma_{t}(\alpha)\right|\right) .
$$

Then $\operatorname{Im}(l)$ is a lattice in $\mathbb{R}^{t}$ of dimension $t-1$ ([17], Theorem 12.5). So we want to find an algebraic unit $\mu \in K$ such that $l_{i}(\mu) \neq 0$ and $l_{j}(\mu) \neq-l_{i}(\mu)$ holds for all $1 \leq i, j \leq n$. However, since $\mu$ is an algebraic unit, we know that $\left|\sigma_{1}(\mu) \ldots \sigma_{t}(\mu)\right|^{2}=1$, which translates to $l_{1}(\mu)+\cdots+l_{t}(\mu)=0$.

Observe that in the case $t=2$ (or $\varphi(d)=4$ ) we can easily see that we cannot find an algebraic unit $\mu \in K$ satisfying the required conditions, since these contradict the fact that we now know that $\log \left|\sigma_{1}(\mu)\right|+\log \left|\sigma_{2}(\mu)\right|=0$. Analogously, the case $t=1$ (or $\varphi(d)=2$ ) can be excluded.

Choose $\varepsilon_{1}, \ldots, \varepsilon_{t-1} \in U_{K}$ such that $\left\{l\left(\varepsilon_{1}\right), \ldots, l\left(\varepsilon_{t-1}\right)\right\}$ generates the lattice $l\left(U_{k}\right)$; in other words,

$$
\operatorname{Im}(l)=\left\{a_{1} l\left(\varepsilon_{1}\right)+\cdots+a_{t-1} l\left(\varepsilon_{t-1}\right) \mid a_{i} \in \mathbb{Z}\right\} .
$$

Our goal is now to see when we can find $a_{i} \in \mathbb{Z}$ such that

$$
a_{1} l\left(\varepsilon_{1}\right)+\cdots+a_{t-1} l\left(\varepsilon_{t-1}\right)=\left(b_{1}, \ldots, b_{t}\right)
$$

with $b_{i} \neq 0$ and $b_{j} \neq-b_{i}$ for all $i, j$.

Let $V$ be the real vector space generated by $\left\{l\left(\varepsilon_{1}\right), \ldots, l\left(\varepsilon_{t-1}\right)\right\}$, then $V \cong \mathbb{R}^{t-1}$. We also know that the subspace of $\mathbb{R}^{t}$ generated by $\operatorname{Im}(l)$ is contained in the hyperplane $\left\{\left(x_{1}, \ldots, x_{t}\right) \in \mathbb{R}^{t} \mid x_{1}+\cdots+x_{t}=0\right\}$, so $V$ will be exactly this hyperplane and

$$
v=(1,2,3, \ldots, t-1,-(1+2+\cdots+t-1)) \in V .
$$

So we can find $r_{1}, \ldots, r_{t-1} \in \mathbb{R}$ such that

$$
r_{1} l\left(\varepsilon_{1}\right)+\cdots+r_{t-1} l\left(\varepsilon_{t-1}\right)=v .
$$

Now if we choose $q_{i} \in \mathbb{Q}$ close enough to $r_{i}$ for all $1 \leq i \leq t-1$, then

$$
\begin{aligned}
v^{\prime} & =q_{1} l\left(\varepsilon_{1}\right)+\cdots+q_{t-1} l\left(\varepsilon_{t-1}\right) \\
& =\left(1+\epsilon_{1}, 2+\epsilon_{2}, \ldots, t-1+\epsilon_{t-1},-\left(1+2+\cdots+t-1+\epsilon_{1}+\cdots+\epsilon_{t-1}\right)\right),
\end{aligned}
$$

with $\epsilon_{i} \in \mathbb{R}$ small, will still have no coefficients $b_{i}, b_{j}$ such that $b_{i}=0$ or $b_{j}=-b_{i}$.

To finish the proof, choose $z \in \mathbb{Z}_{0}$ such that $z q_{i} \in \mathbb{Z}$ for all $1 \leq i \leq t-1$, and let $a_{i}=z q_{i}$ for all $i$. Then these are exactly the $a_{i}$ we were looking for, and $\mu=\varepsilon_{1}^{a_{1}} \ldots \varepsilon_{t-1}^{a_{t-1}}$ will satisfy the conditions of the lemma.

Furthermore, because we want our commuting matrix $C$ to be in $\operatorname{GL}(n, \mathbb{Q})$, we will need the following number theoretical theorem later on: 
Theorem 6.5. Let $K=\mathbb{Q}(\theta)$ be an algebraic number field and $\left\{\sigma_{1}, \ldots, \sigma_{n}\right\}$ the monomorphisms from $K$ to $\mathbb{C}$. If $P\left(X_{1}, \ldots, X_{n}\right)$ is a symmetric polynomial over $\mathbb{Q}$ and $\alpha \in K$, then $P\left(\sigma_{1}(\alpha), \ldots, \sigma_{n}(\alpha)\right) \in \mathbb{Q}$.

We can now formulate the next theorem, which reduces the conditions on the $\mathbb{Q}$-irreducible components of $T$ of multiplicity one in Theorem 6.1.

Theorem 6.6. Let $T: F \rightarrow \mathrm{GL}(n, \mathbb{Q})$ be a $\mathbb{Q}$-irreducible representation of a finite, abelian group $F$ and write $\Phi=\operatorname{Im}(T)$. Then the following assertions are equivalent:

(1) There exists a matrix $C \in \operatorname{GL}(n, \mathbb{Q})$ that commutes with every element of $\Phi$, and such that $C$ has a normalized integer characteristic polynomial which has no roots $\lambda$, $\mu$ with $|\lambda|=1$ or $|\lambda \mu|=1$.

(2) $|\Phi|>6$ and $|\Phi| \neq 8,10$ and 12 .

Proof. Choose a generator $M$ of $\Phi$ and write $d=|\Phi|$, so $d$ is the order of $M$. Let $\xi_{d}$ be a primitive $d$-th root of unity and write $K=\mathbb{Q}\left(\xi_{d}\right)$ and $\Gamma(K / \mathbb{Q})=$ $\left\{\sigma_{1}, \ldots, \sigma_{\varphi(d)}\right\}$. Then $\sigma_{1}\left(\xi_{d}\right), \ldots, \sigma_{\varphi(d)}\left(\xi_{d}\right)$ are the eigenvalues of $M$ and we can find a basis $\left\{\sigma_{1}(v), \ldots, \sigma_{\varphi(d)}(v)\right\}$ of $K^{n}$ over $K$ in which every $\sigma_{i}(v)$ is an eigenvector of $M$ corresponding to the eigenvalue $\sigma_{i}\left(\xi_{d}\right)$.

Suppose first that $|\Phi|>6$ and $|\Phi| \neq 8,10$ and 12. If we write $d=p_{1}^{e_{1}} p_{2}^{e_{2}} \ldots p_{r}^{e_{r}}$, with $r \geq 1, p_{i}$ pairwise different primes and $e_{i} \in \mathbb{N}$ for all $i$, then $\varphi(d)=$ $\left(p_{1}-1\right) p_{1}^{e_{1}-1}\left(p_{2}-1\right) p_{2}^{e_{2}-1} \ldots\left(p_{r}-1\right) p_{r}^{e_{r}-1}$, so for the chosen values of $|\Phi|$ we have that $\varphi(d) \geq 6$. According to the lemma above, we can find an algebraic unit $\mu \in U_{K}$ satisfying $\left|\sigma_{i}(\mu)\right| \neq 1$ and $\left|\sigma_{i}(\mu) \sigma_{j}(\mu)\right| \neq 1$ for all $1 \leq i, j \leq \varphi(d)$. Now let

$$
\widetilde{C}=\left(\begin{array}{ccc}
\sigma_{1}(\mu) & \cdots & 0 \\
\vdots & \ddots & \vdots \\
0 & \cdots & \sigma_{\varphi(d)}(\mu)
\end{array}\right) \in \operatorname{GL}(n, K)
$$

be the diagonal matrix with the $\sigma_{i}(\mu)$ on the diagonal. Then $\widetilde{C}$ commutes with $P^{-1} M P$, so if we take $C=P \widetilde{C} P^{-1}$, then $C$ commutes with $M$. Since $C$ and $\widetilde{C}$ have the same characteristic polynomial and the same eigenvalues, $\operatorname{Spec}(C)=$ $\left\{\sigma_{1}(\mu), \ldots, \sigma_{\varphi(d)}(\mu)\right\}$, so the eigenvalues of $C$ satisfy the conditions by construction. Also, since $\mu$ is an algebraic unit, the constant term of the characteristic polynomial is \pm 1 . By Theorem 6.5 , it is also easy to see that the characteristic polynomial of $C$ has rational coefficients; but since those coefficients are also algebraic integers and the only algebraic integers in $\mathbb{Q}$ are the elements of $\mathbb{Z}$, the coefficients of the characteristic polynomial will be integers.

The only thing that is left to show now is that $C \in \mathbb{Q}^{n \times n}$. Consider the linear transformation $h: K^{n} \rightarrow K^{n}$ that has matrix representation $C$ with respect to the standard basis; thus, with respect to the basis $\left\{\sigma_{1}(v), \ldots, \sigma_{\varphi(d)}(v)\right\}$ it has matrix representation $\widetilde{C}$. We will show that $C \in \mathbb{Q}^{n \times n}$ by showing that $h\left(e_{j}\right) \in \mathbb{Q}^{n}$ for every $j$. 
For $1 \leq j \leq n$, we can write

$$
e_{j}=a_{1}^{j} \sigma_{1}(v)+\cdots+a_{n}^{j} \sigma_{n}(v)
$$

for unique $a_{i}^{j} \in K, 1 \leq i \leq n$. But then, for $1 \leq k \leq \varphi(d)$, we also have

$$
\sigma_{k}\left(e_{j}\right)=\sigma_{k}\left(a_{1}^{j} \sigma_{1}(v)\right)+\cdots+\sigma_{k}\left(a_{n}^{j} \sigma_{n}(v)\right)
$$

or, equivalently,

$$
e_{j}=\sigma_{k}\left(a_{1}^{j}\right)\left(\sigma_{k} \circ \sigma_{1}\right)(v)+\cdots+\sigma_{k}\left(a_{n}^{j}\right)\left(\sigma_{k} \circ \sigma_{n}\right)(v)
$$

since $e_{j} \in \mathbb{Q}^{n}$. We may suppose that $\sigma_{1}=\mathrm{Id}$. Then by the uniqueness of the $a_{i}^{j}$, we can see that $a_{k}^{j}=\sigma_{k}\left(a_{1}^{j}\right)$ for every $k$, so we can find $a^{j} \in K$ such that

$$
e_{j}=\sigma_{1}\left(a^{j} v\right)+\cdots+\sigma_{n}\left(a^{j} v\right) .
$$

But then

$$
\begin{aligned}
h\left(e_{j}\right) & =\sigma_{1}\left(a^{j}\right) h\left(\sigma_{1}(v)\right)+\cdots+\sigma_{n}\left(a^{j}\right) h\left(\sigma_{n}(v)\right) \\
& =\sigma_{1}\left(a^{j}\right) \sigma_{1}(\mu) \sigma_{1}(v)+\cdots+\sigma_{n}\left(a^{j}\right) \sigma_{n}(\mu) \sigma_{n}(v) \\
& =\sigma_{1}\left(a^{j} \mu v\right)+\cdots+\sigma_{n}\left(a^{j} \mu v\right),
\end{aligned}
$$

which is a symmetric polynomial evaluated in the $\sigma_{i}\left(a^{j} \mu v\right)$ and so $h\left(e_{j}\right) \in \mathbb{Q}^{n}$ by Theorem 6.5.

Conversely, suppose that $|\Phi| \leq 6$ or $|\Phi|=8,10$ or 12 and that $C \in \operatorname{GL}(n, \mathbb{Q})$ commutes with $M$. Then $\varphi(d) \leq 4$ and we can easily see by Lemma 6.4 that the characteristic polynomial of $C$ cannot satisfy all conditions.

Remark 6.7. The property $|\Phi|>6$ and $|\Phi| \neq 8,10$ and 12 of the above theorem is also equivalent to each of the following statements:

(1) The dimension $n$ of the irreducible representation $T: F \rightarrow \operatorname{GL}(n, \mathbb{Q})$ is at least 6.

(2) The $\mathbb{Q}$-irreducible representation $T: F \rightarrow \operatorname{GL}(n, \mathbb{Q})$ splits in at least three components when seen as a representation $T: F \rightarrow \operatorname{GL}(n, \mathbb{R})$ over $\mathbb{R}$.

We want to do something similar for the $\mathbb{Q}$-irreducible components of multiplicity two.

Theorem 6.8. Let $T_{1}: F \rightarrow \operatorname{GL}(n, \mathbb{Q})$ be a $\mathbb{Q}$-irreducible representation of a finite, abelian group $F$, and write $T=T_{1} \oplus T_{1}: F \rightarrow \operatorname{GL}(2 n, \mathbb{Q})$ and $\Phi=\operatorname{Im}(T)$. Then the following assertions are equivalent: 
(1) There exists a matrix $C \in \mathrm{GL}(2 n, \mathbb{Q})$ commuting with every element of $\Phi$ such that $C$ has a normalized integer characteristic polynomial which has no roots $\lambda, \mu$ with $|\lambda|=1$ or $|\lambda \mu|=1$.

(2) $|\Phi|>4$ and $|\Phi| \neq 6$.

Proof. Let $M=\left(\begin{array}{cc}M_{1} & 0_{n} \\ 0_{n} & M_{1}\end{array}\right)$ be a generator of $\Phi$ with $M_{1} \in \mathrm{GL}(n, \mathbb{Q})$. First suppose that $|\Phi|>4$ and $|\Phi| \neq 6$. If $|\Phi|>6$ and $|\Phi| \neq 8,10$ and 12 , we can find $C_{1} \in \mathrm{GL}(n, \mathbb{Q})$ commuting with $M_{1}$ whose characteristic polynomial satisfies the conditions, so $C=\left(\begin{array}{ll}C_{1} & 0_{n} \\ 0_{n} & C_{1}\end{array}\right) \in \operatorname{GL}(2 n, \mathbb{Q})$ commutes with $M$. Thus we only have to take a closer look to the case $|\Phi|=5,8,10$ or 12 . Let $\xi_{d}$ be an eigenvalue of $M_{1}$, with $d=5,8,10$ or 12 . Then $\varphi(d)=4$ and we can write $K=\mathbb{Q}\left(\xi_{d}\right)$ to be the splitting field of $T_{1}$ and $\Gamma(K / \mathbb{Q})=\left\{\sigma_{1}, \sigma_{2}, \sigma_{3}, \sigma_{4}\right\}$. Now $M_{1} \in \mathrm{GL}(4, \mathbb{Q})$ and in the same way as before, we can find $P_{1} \in \mathrm{GL}(4, K)$ such that

$$
P_{1}^{-1} M_{1} P_{1}=\left(\begin{array}{cccc}
\sigma_{1}\left(\xi_{d}\right) & 0 & 0 & 0 \\
0 & \sigma_{2}\left(\xi_{d}\right) & 0 & 0 \\
0 & 0 & \sigma_{3}\left(\xi_{d}\right) & 0 \\
0 & 0 & 0 & \sigma_{4}\left(\xi_{d}\right)
\end{array}\right)
$$

Write $K=\mathbb{Q}\left(e^{\frac{\Pi i}{m}}\right)$ (you can always do this) and take $L=\mathbb{Q}\left(e^{\frac{\Pi i}{2 m}}\right)$. Then $L$ is a splitting field of the polynomial $X^{2}-e^{\frac{\Pi i}{m}}$ over $K, L$ is Galois over $K$ and $\Gamma(L / K)=\left\{s_{1}, s_{2}\right\}$ (suppose that $s_{1}\left(e^{\frac{\Pi i}{2 m}}\right)=e^{\frac{\Pi i}{2 m}}$ and $\left.s_{2}\left(e^{\frac{\Pi i}{2 m}}\right)=e^{\frac{-\Pi i}{2 m}}\right)$. We can write $\Gamma(L / \mathbb{Q})=\left\{\eta_{1}, \ldots, \eta_{8}\right\}$ and we may suppose, without loosing generality, that $s_{1}=\eta_{1}$ and $s_{2}=\eta_{2}$. By Lemma 6.4, we can now choose an algebraic unit $\mu$ in $L$ for which $\left|\eta_{i}(\mu)\right| \neq 1$ and $\left|\eta_{i}(\mu) \eta_{j}(\mu)\right| \neq 1$ for all $1 \leq i, j \leq 8$ ( $\mu$ will certainly not be in $K)$. Now $\eta_{1}(\mu) \eta_{2}(\mu) \in K$ and $\eta_{1}(\mu)+\eta_{2}(\mu) \in K$ since they are invariant under the morphisms of $\Gamma(L / K)$. So $\eta_{1}(\mu)$ and $\eta_{2}(\mu)$ are roots of

$$
f(X)=X^{2}-\left(\eta_{1}(\mu)+\eta_{2}(\mu)\right) X+\eta_{1}(\mu) \eta_{2}(\mu) \in K[X] .
$$

Now consider the polynomials

$$
m_{i}(X)=\sigma_{i}(f(X)), 1 \leq i \leq 4 .
$$

Then

$$
m(X)=m_{1}(X) m_{2}(X) m_{3}(X) m_{4}(X) \in \mathbb{Q}[X]
$$

and $\eta_{1}(\mu)$ is a root of this polynomial, so all Galois-conjugates of $\mu$ are also roots. This means that $m(X)$ is (a power of) the monic minimal polynomial of $\mu$ over $\mathbb{Q}$; but since $\mu$ is an algebraic integer, this is a polynomial over $\mathbb{Z}$. Now suppose that $\eta_{1}(\mu)$ and $\eta_{2}(\mu)$ are the roots of $m_{1}(X), \eta_{3}(\mu)$ and $\eta_{4}(\mu)$ are the roots of $m_{2}(X), \eta_{5}(\mu)$ and $\eta_{6}(\mu)$ are the roots of $m_{3}(X)$, and $\eta_{7}(\mu)$ and $\eta_{8}(\mu)$ are the roots of $m_{4}(X)$. Choose

$$
\widetilde{C}=\left(\begin{array}{cc}
0_{4} & -1_{4} \\
M_{\times} & M_{+}
\end{array}\right)
$$


in which

$$
M_{\times}=\left(\begin{array}{cccc}
\eta_{1}(\mu) \eta_{2}(\mu) & 0 & 0 & 0 \\
0 & \eta_{3}(\mu) \eta_{4}(\mu) & 0 & 0 \\
0 & 0 & \eta_{5}(\mu) \eta_{6}(\mu) & 0 \\
0 & 0 & 0 & \eta_{7}(\mu) \eta_{8}(\mu)
\end{array}\right)
$$

and

$$
M_{+}=\left(\begin{array}{cccc}
\eta_{1}(\mu)+\eta_{2}(\mu) & 0 & 0 & 0 \\
0 & \eta_{3}(\mu)+\eta_{4}(\mu) & 0 & 0 \\
0 & 0 & \eta_{5}(\mu)+\eta_{6}(\mu) & 0 \\
0 & 0 & 0 & \eta_{7}(\mu)+\eta_{8}(\mu)
\end{array}\right) \text {. }
$$

The characteristic polynomial of $\widetilde{C}$ is exactly $m(X)$ and so, by construction, the characteristic polynomial of $\widetilde{C}$ satisfies the conditions and $\widetilde{C}$ commutes with $M$. Now consider the matrix $P_{1} \in \mathrm{GL}(4, K)$ as before and let $P=\left(\begin{array}{ll}P_{1} & 0_{4} \\ 0_{4} & P_{1}\end{array}\right)$. Then

$$
P^{-1} \tilde{C} P=\left(\begin{array}{cc}
0_{4} & -1_{4} \\
P_{1}^{-1} M_{\times} P_{1} & P_{1}^{-1} M_{+} P_{1}
\end{array}\right)
$$

will still satisfy the conditions on the characteristic polynomial and will commute with $M$. In the same way as in the proof of Theorem 6.6, we can now show that $C \in \mathbb{Q}^{8 \times 8}$.

Conversely, suppose that $|\Phi| \leq 4$ or $|\Phi|=6$. Then $M \in \operatorname{GL}(2, \mathbb{Q})$ resp. $\mathrm{GL}(4, \mathbb{Q})$, and it is easy to see that there is no $C \in \mathrm{GL}(2, \mathbb{Q})$ resp. $\operatorname{GL}(4, \mathbb{Q})$, commuting with $M$, whose characteristic polynomial satisfies the conditions.

Remark 6.9. The property $|\Phi|>4$ and $|\Phi| \neq 6$ of the above theorem is also equivalent to each of the following statements:

(1) The dimension $n$ of the irreducible representation $T: F \rightarrow \mathrm{GL}(n, \mathbb{Q})$ is at least 4.

(2) The $\mathbb{Q}$-irreducible representation $T: F \rightarrow \mathrm{GL}(n, \mathbb{Q})$ is reducible, when seen as a representation $T: F \rightarrow \mathrm{GL}(n, \mathbb{R})$ over $\mathbb{R}$.

\section{Main theorem}

As a consequence of what we have done so far, we obtain the main theorem of this article.

Theorem 7.1. Let $M$ be an infra-nilmanifold modeled on a free 2-step nilpotent Lie group, with abelian holonomy group $F$ and associated abelianized rational holonomy representation $\bar{\varphi}: F \rightarrow \operatorname{Aut}\left(\frac{N_{\mathbb{Q}}}{\left[N_{\mathbb{Q}}, N_{\mathbb{Q}}\right]}\right)$. Then $M$ admits an Anosov diffeomorphism if and only if 
(1) for every $\mathbb{Q}$-irreducible component $\bar{\varphi}_{i}$ of $\bar{\varphi}$ of multiplicity one, we have $\left|\operatorname{Im}\left(\bar{\varphi}_{i}\right)\right|>6$ and $\left|\operatorname{Im}\left(\bar{\varphi}_{i}\right)\right| \neq 8,10$ and $12 ;$

(2) for every $\mathbb{Q}$-irreducible component $\bar{\varphi}_{i}$ of $\bar{\varphi}$ of multiplicity two, we have $\left|\operatorname{Im}\left(\bar{\varphi}_{i}\right)\right|>4$ and $\left|\operatorname{Im}\left(\bar{\varphi}_{i}\right)\right| \neq 6$.

Proof. By using Theorem 6.1, we can reduce Theorem B, for this type of infranilmanifolds, to the following:

$M$ admits an Anosov diffeomorphism if and only if for every $\mathbb{Q}$-irreducible component $\bar{\varphi}_{i}: F \rightarrow \operatorname{Aut}\left(V_{i}\right)$ that occurs with multiplicity one resp. two (where $V_{i}$ is an $n_{i}$-dimensional subspace of $\left.\frac{N_{\mathbb{Q}}}{\left[N_{\mathbb{Q}}, N_{\mathbb{Q}}\right]}\right)$, there exists an automorphism $\bar{\psi}_{i} \in \operatorname{Aut}\left(V_{i}\right)$ resp. $\bar{\psi}_{i} \in \operatorname{Aut}\left(V_{i} \oplus V_{i+1}\right)$ (with $\left.V_{i+1} \cong V_{i}\right)$ such that

(1) $\bar{\psi}_{i}$ has a normalized integer characteristic polynomial,

(2) $\bar{\psi}_{i}$ commutes with any element of $\bar{\varphi}_{i}(F)$ resp. $\left(\bar{\varphi}_{i} \oplus \bar{\varphi}_{i}\right)(F)$,

(3) $\bar{\psi}_{i}$ has no eigenvalues $\lambda, \mu$ with $|\lambda|=1$ or $|\lambda \mu|=1$.

So we only have to find out when we can find an automorphism commuting with the $\mathbb{Q}$-irreducible components of $\bar{\varphi}$ occurring with multiplicity one or two. By using Theorem 6.6 and Theorem 6.8, we get exactly the conditions stated in the theorem.

In order to compare this theorem with the original result of Porteous mentioned before, we use the Remarks 6.7 and 6.9 and formulate the above result as Theorem $\mathrm{C}$ from the introduction.

This result and some more experiments we have carried out lead to the following conjecture

Conjecture 7.2. Let $M$ be an infra-nilmanifold modeled on a free c-step nilpotent Lie group, with holonomy group $F$ and associated abelianized rational holonomy representation $\bar{\varphi}: F \rightarrow \operatorname{Aut}\left(\frac{N_{\mathbb{Q}}}{\left[N_{\mathbb{Q}}, N_{\mathbb{Q}}\right]}\right)$. Then $M$ admits an Anosov diffeomorphism if and only if each $\mathbb{Q}$-irreducible component $\bar{\varphi}_{i}$ of $\bar{\varphi}$ of multiplicity $m$ splits in at least $c+2-m$ components when seen as a representation over $\mathbb{R}$.

\section{References}

[1] S. G. Dani, Nilmanifolds with Anosov automorphism. J. London Math. Soc. (2) 18 (1978), 553-559. Zbl 0399.58006 MR 518242

[2] S. G. Dani and M. G. Mainkar, Anosov automorphisms on compact nilmanifolds associated with graphs. Trans. Amer. Math. Soc. 357 (2005), 2235-2251. Zbl 1061.22008 MR 2140439

[3] K. Dekimpe, Almost-Bieberbach groups: affine and polynomial structures. Lecture Notes in Math. 1639, Springer-Verlag, Berlin 1996. Zbl 0865.20001 MR 1482520 
[4] K. Dekimpe, Hyperbolic automorphisms and Anosov diffeomorphisms on nilmanifolds. Trans. Amer. Math. Soc. 353 (2001), 2859-2877. Zbl 0990.37022 MR 1828476

[5] K. Dekimpe and K. B. Lee, Expanding maps on infra-nilmanifolds of homogeneous type. Trans. Amer. Math. Soc. 355 (2003), 1067-1077. Zbl 1009.37018 MR 1938746

[6] J. Franks, Anosov diffeomorphisms. In Global analysis (Berkeley, Calif., 1968), Proc. Sympos. Pure Math. 14, Amer. Math. Soc., Providence, R.I., 1970, 61-93.

Zbl 0207.54304 MR 0271990

[7] M. W. Hirsch, Anosov maps, polycyclic groups, and homology. Topology 10 (1971), 177-183. Zbl 0211.26801 MR 0283824

[8] J. Lauret, Examples of Anosov diffeomorphisms. J. Algebra 262 (2003), 201-209. Zbl 1015.37022 MR 1970807

[9] K. B. Lee, Infra-solvmanifolds of type (R). Quart. J. Math. Oxford Ser. (2) 46 (1995), 185-195. Zbl 0857.22009 MR 1333830

[10] K. B. Lee, Maps on infra-nilmanifolds - Rigidity and applications to fixed-point theory. Pacific J. Math. 168 (1995), 157-166. Zbl 0920.55003 MR 1331996

[11] K. B. Lee and F. Raymond, Rigidity of almost crystallographic groups. In Combinatorial methods in topology and algebraic geometry (Rochester, N.Y., 1982), Contemp. Math. 44, Amer. Math. Soc., Providence, RI, 1985, 73-78. Zbl 0575.57026 MR 0813102

[12] M. G. Mainkar, Anosov automorphisms on certain classes of nilmanifolds. Glasgow Math. J. 48 (2006), 161-170. Zbl 1134.37325 MR 2224936

[13] M. G. Mainkar and C. E. Will, Examples of Anosov Lie algebras. Discrete Contin. Dyn. Syst. 18 (2007), 39-52. Zbl 1120.37014 MR 2276485

[14] A. Manning, There are no new Anosov diffeomorphisms on tori. Amer. J. Math. 96 (1974), 422-429. Zbl 0242.58003 MR 0358865

[15] H. L. Porteous, Anosov diffeomorphisms of flat manifolds. Topology 11 (1972), 307-315. Zbl 0237.58015 MR 0296976

[16] D. Segal, Polycyclic groups. Cambridge Tracts in Math. 82, Cambridge University Press, Cambridge 1983. Zbl 0516.20001 MR 0713786

[17] I. Stewart and D. Tall, Algebraic number theory. 2nd ed., Chapman and Hall Mathematics Series, Chapman \& Hall, London 1987. Zbl 0663.12001 MR 0896691

Received July 20, 2007; revised July 16, 2008

K. Dekimpe and K. Verheyen, K.U.Leuven Campus Kortrijk, Universitaire campus, 8500 Kortrijk, Belgium

E-mail: Karel.Dekimpe@kuleuven-kortrijk.be, kelly.verheyen@kuleuven-kortrijk.be 